\title{
Three-dimensional earthward fast flow in the near-Earth plasma sheet in a sheared field: comparisons between simulations and observations
}

\author{
K. Kondoh and M. Ugai \\ Research Center for Space and Cosmic Evolution, Ehime University, Japan
}

Received: 20 October 2008 - Revised: 22 April 2009 - Accepted: 24 May 2009 - Published: 8 June 2009

\begin{abstract}
Three-dimensional configuration of earthward fast flow in the near-Earth plasma sheet is studied using threedimensional magnetohydrodynamics (MHD) simulations on the basis of the spontaneous fast reconnection model. In this study, the sheared magnetic field in the plasma sheet is newly considered in order to investigate the effects of it to the earthward fast flow, and the results are discussed in comparison with no-shear simulations. The virtual probes located at different positions in our simulation domain in shear/no-shear cases could explain different behavior of fast flows in the real observations.
\end{abstract}

Keywords. Magnetospheric physics (Plasma sheet) - Space plasma physics (Magnetic reconnection; Numerical simulation studies)

\section{Introduction}

The existence of earthward fast flow events, which are called Bursty Bulk Flows (BBFs) or Flow Bursts (FBs) (Angelopoulos et al., 1992), in the near-Earth plasma sheet during active magnetospheric conditions have been pointed out by a lot of individual case studies (Sergeev et al., 2000, 2001; Angelopoulos et al., 1992). These previous studies have shown that earthward BBFs are generally accompanied by dipolarization of the plasma sheet, and BBFs are directed either earthward or tailward. BBFs are, therefore, often considered to be signatures of bursty and localized reconnection in the plasma sheet (Ohtani et al., 2004; Schodel et al., 2001).

Magnetic reconnection can convert magnetic energy to plasma energies most effectively by slow shocks. We have

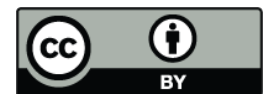

Correspondence to: $\mathrm{K}$. Kondoh (kondo@ cosmos.ehime-u.ac.jp) proposed the spontaneous fast reconnection model. This model predicts that the fast reconnection mechanism spontaneously develops from inside the system by the selfconsistent interaction between plasma microscopic processes and macroscopic reconnection flows (Ugai, 1984, 1986). The previous magnetohydrodynamics (MHD) simulations have demonstrated that the spontaneous fast reconnection model works quite effectively even in three-dimensional situations (Ugai and Kondoh, 2001; Ugai et al., 2004, 2005). Once the fast reconnection builds up, an Alfvénic fast flow is caused, and if the fast flow is obstructed, the reconnected field lines are piled up, and a magnetic loop is formed. A magnetic loop in our simulations is allowed to be formed because of a wall boundary assumed at one edge of a current sheet system (Ugai et al., 2003).

In the previous study, we showed that virtual probe observations, which were located in our simulation domain, consist with superposed epoch analysis of actual in-situ GEOTAIL satellite observation data (Kondoh and Ugai, 2008). However, the time variations observed by actual satellites are quite different in each observation. Figure 1 shows the time variations of earthward component of flow velocity $\left(V_{x}\right)$, magnetic field components $\left(B_{x}, B_{y}, B_{z}\right)$ and plasma density $(N)$ in the GSM coordinate system in the two sample earthward fast flow events observed by GEOTAIL satellite in 23 January 1997 (a) and 26 November 1997 (b). In the left event (a), the long enhancement of $V_{x}$ and the rapid decrease of $N$ are observed. On the other hand, the rapid decrease of $V_{x}$ and the gradual small decrease of $N$ are observed in the right event (b). These differences are usually interpreted by the position of the satellite without considering the magnetic field structure. Here, we should notice the variation of magnetic field components, especially $B_{y}$. In the event (b), the large shear field component $B_{y}$ is observed before observing the fast flow event. In this study, we focus to the effects of the shear field $B_{y}$ on the fast reconnection evolution so as

Published by Copernicus Publications on behalf of the European Geosciences Union. 

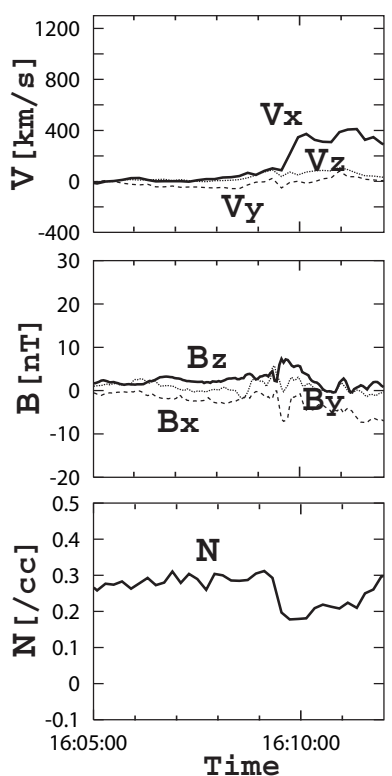

(a) Jan. 23, 1997(-30.40, 2.83, -2.56) (b)Nov. 26, 1997(-18.82, 0.72, -2.01)

Fig. 1. Two sample events of earthward fast plasma flow observed by GEOTAIL satellite in 23 January 1997 (a) and 26 November 1997 (b).

to predict the satellite position. The 3-D fast reconnection evolution in a shear field has not been studied for detailed applications to the BBF observations.

\section{Simulation model}

As mentioned in the introduction, the purpose of this study is to investigate the effects of the shear field component in the plasma sheet on the earthward fast flow event. Hence, the simulation domain is extended to the second quadrant whereas in the previous studies only the first quadrant is considered (Kondoh and Ugai, 2008). As shown in Fig. 2, the axis symmetry boundary conditions are adopted in order to consider the shear field component.

\subsection{Simulation modeling}

Simulation model in this study is the same as that in the previous simulations (Kondoh and Ugai, 2008) except for the above boundary conditions. Our previous magnetic loop simulations using the wall boundary showed that the fast magnetic reconnection produces fast reconnection jet, and it flows in plasma sheet and is suddenly braked at the boundary between the dipolar and tail-like magnetic field because of the counterward pressure force, and the reconnection jet is decelerated (Ugai et al., 2003). This situation may be consistent with the earthward flow, and these results are in good agreement with the situation inferred from the observation results of earthward flow (Shiokawa et al., 1997).

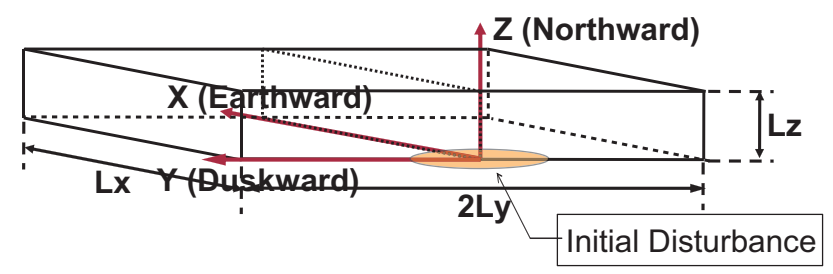

Fig. 2. Computation region and the coordinate system adopted in our simulations.

\subsubsection{Initial-boundary-value problem}

As an initial configuration, the anti-parallel magnetic field $\mathbf{B}=\left[B_{x}(z), B_{y 0}, 0\right]$ is assumed as: $B_{x}(z)=\sin (\pi z / 2)$ for $0<z<1 ; B_{x}(z)=1$ for $1<z<Z_{1} ; B_{x}(z)=\cos \left[\left(z-Z_{1}\right) \pi / 1.2\right]$ for $Z_{1}<z<Z_{m}\left(=Z_{1}+0.6\right) ; \quad B_{x}(z)=0$ for $Z_{m}<z$; also, $B_{x}(z)=-B_{x}(-z)$ for $z<0$. The shear magnetic field component $B_{y 0}$ is considered to be 0.2 in this study. The plasma pressure $P(z)$ initially satisfies the pressure-balance condition,

$P+B_{x}^{2}=1+\beta_{0}$

where $\beta_{0}$ is the ratio of plasma pressure to the magnetic pressure in the ambient magnetic field region $1<z<Z_{1}$, so that $P(z=0)=1+\beta_{0}$ initially (in the present study, $\beta_{0}=0.15$ is taken); Initially, fluid velocity $\mathbf{u}=(0,0,0)$, and, constant temperature $T=P / \rho=1+\beta_{0}$ is assumed, so that the plasma density $\rho$ initially satisfies

$\rho(z)=P(z) /\left(1+\beta_{0}\right)$,

In our previous studies, a number of computations have been done for different plasma $\beta \mathrm{s}$ and temperatures to examine these effects on the fast reconnection evolution (Ugai, 1992, 1999; Ugai and Zheng, 2006). The normalization of quantities, based on the initial quantities, is self-evident; Distances are normalized by the half-width of the current sheet $d_{0}, \mathbf{B}$ by the field strength in the magnetic field region $B_{x 0}, P$ by $B_{x 0}^{2} /\left(2 \mu_{0}\right)$, and $\rho$ by $\rho_{i}=\rho(z=0)$; also, $\mathbf{u}$ by $V_{A x 0}\left(=B_{x 0} / \sqrt{\mu_{0} \rho_{i}}\right)$, time $t$ by $d_{0} / V_{A x 0}$, current density $\mathbf{J}$ by $J_{0}=B_{x 0} /\left(\mu_{0} d_{0}\right)$, and so forth. Note that the Alfvén velocity in the ambient magnetic field region $\left(1<z<Z_{1}\right)$ at initial state is given by $V_{A e}=V_{A x 0} / \sqrt{\rho_{e}}\left(\rho_{e}\right.$ is the density there).

Here, the conventional axis symmetry boundary conditions are assumed on the $(x, y)$, and $(y, z)$ planes. Hence, the computational region can be restricted to the first and second quadrants and taken to be a rectangular box, $0<x<L_{x}$, $-L_{y}<y<L_{y}$, and $0<z<L_{z}$; also, for simplicity, the conventional symmetry boundary condition is assumed on the outer boundary plane $x=L_{x}$, and on the other boundary planes $\left(y=-L_{y}, L_{y}\right.$ and $\left.z=L_{z}\right)$ the free boundary conditions are assumed.

Current-driven anomalous resistivity have been studied theoretically and experimentally (Lui, 2001; Treumann, 
2001; Ono et al., 2001; Petkaki and Freeman, 2008). Here, as in the 2-D model, the anomalous resistivity model is assumed in the form,

$$
\begin{aligned}
\eta(\mathbf{r}, t) & =k_{R}\left[V_{d}(\mathbf{r}, t)-V_{C}\right] & & \text { for } V_{d}>V_{C}, \\
& =0 & & \text { for } V_{d}<V_{C}
\end{aligned}
$$

where $V_{d}(\mathbf{r}, t)=|\mathbf{J}(\mathbf{r}, t) / \rho(\mathbf{r}, t)|$ is the relative electron-ion drift velocity, and $V_{C}$ may be a threshold for microinstabilities. Here, $k_{R}=0.003$ and $V_{C}=12$ are taken (Ugai, 1984, 1992, 1999).

In order to disturb the initial static configuration, a localized resistivity model is assumed around the origin in the 3-D form,

$$
\eta(\mathbf{r})=\eta_{0} \exp \left[-\left(x / k_{x}\right)^{2}-\left(y / k_{y}\right)^{4}-\left(|z| / k_{z}\right)^{3}\right]
$$

Here, we take $k_{x}=k_{z}=0.8$ and $\eta_{0}=0.02$ in the manner similar to the previous 2-D simulations. Also, $k_{y}$ provides the 3-D effects. Previous studies showed that the $k_{y}$ specifies the effective extent of diffusion region in the y-direction, and magnetic reconnection cannot effectively grow in the case of small $k_{y}, k_{y}<3$ say (Kondoh et al., 2006), so that $k_{y}=8$ is taken in the present study.

The disturbance (4) is imposed only in the initial time range $0<t<4$, and the anomalous resistivity model (3) is assumed for $t>4$. Hence, the fast reconnection mechanism may be triggered at $x=0$ in this model.

It should be noted that sufficiently small mesh sizes are required for precise computations of the spontaneous fast reconnection evolution especially in the z-direction, so that we assume $\Delta x=0.04, \Delta y=0.2$, and $\Delta z=0.015$. Also, we take the magnetic field region size $Z_{1}=4$, and the whole computational region size is assumed to be $L_{x}=14, L_{y}=20$ and $L_{z}=9.6$.

\section{Results}

At first, let us show the general results of present 3-D MHD simulations. Initiated by the disturbance (4) in the finite extent $|y| \leq 8$, the current sheet thinning occurs near the origin, giving rise to distinct enhancement of the current density. Once the drift velocity $V_{d}$ exceeds the threshold $V_{c}$ (Eq. 3), fast reconnection drastically grows as a nonlinear instability because of the positive feedback between the anomalous resistivity and the reconnection flow (Ugai, 1992, 1999): that is, the reconnection flow grows so as to enhance the currentdriven anomalous resistivity, which in turn enhance the reconnection flow. Ahead of the Alfvénic reconnection jet flowing to $\mathrm{x}$-direction, a large-scale 3-D plasmoid is formed and propagates, and the plasmoid collides with the $x=L_{x}$ wall boundary, giving rise to a 3-D magnetic loop.

Figure 3 shows 3-D configurations of high pressure region (dark gray (red) iso-surface, $P=1.4$ ) and earthward fast flow (light gray (blue) iso-surface, $V_{x}=1.4$ ) at time $T=39$ just before the plasmoid collides with $x=L_{x}$ wall boundary. High

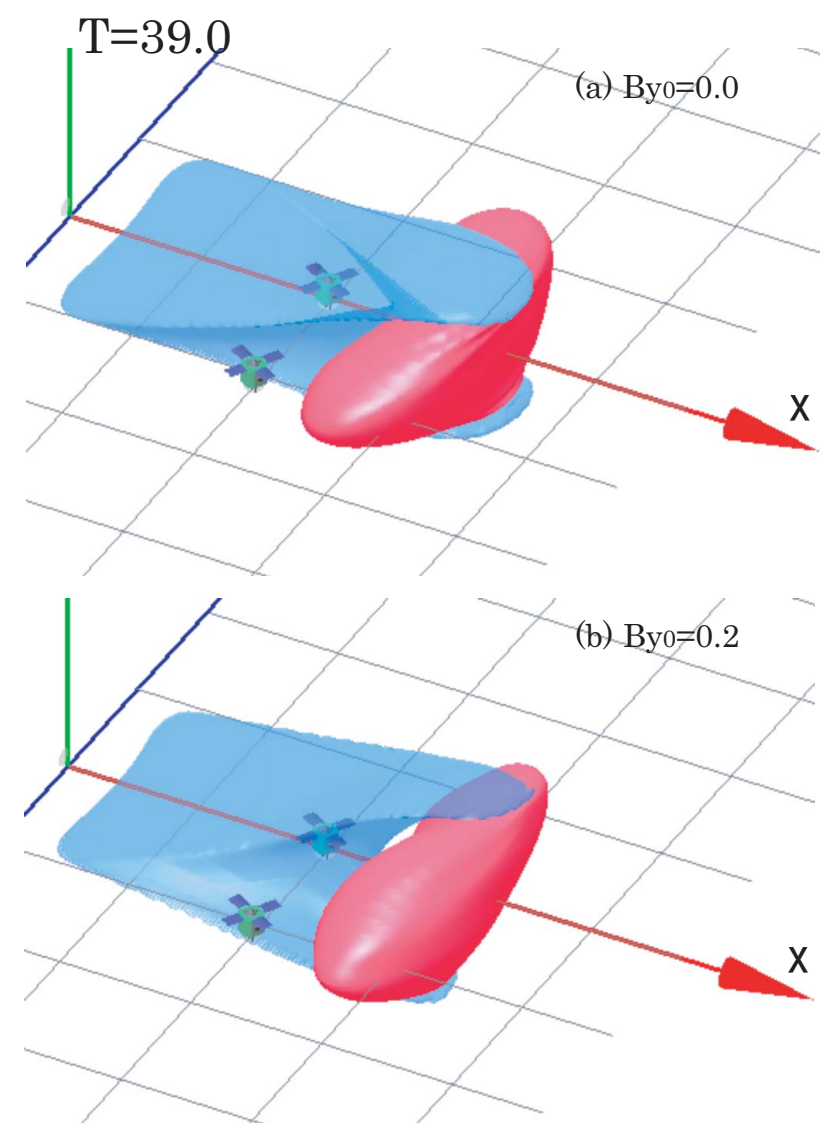

Fig. 3. Isosurfaces of high pressure region (dark gray (red) ones, $P=1.4$ ) and earthward plasma speed (light gray (blue) ones, $V_{x}=1.4$ ) in the two cases of $B_{y 0}=0.0$ (a) and $B_{y 0}=0.2$ (b) at $T=39.0$ derived by our MHD simulations.

pressure region propagates in the $\mathrm{x}$-direction and swells. As can be seen, the thickness of the fast flow region in the zdirection is thin near the reconnection region because of the pinch effect (Ugai, 1992). The top panel (a) shows those in the case of no initial shear field $B_{y 0}=0$, whereas the bottom panel (b) shows those in the case of the initial shear filed $B_{y 0}=0.2$. In the case (b), the fast flow channel deflects in the direction of the shear field compared with that in the case (a), although the width of fast flow channel and high pressure region is almost same in the both cases. The deflection angle of the fast flow is largest at the edge of the fast flow channel in the negative $\mathrm{y}$-direction, and that angle is almost $\arctan \left(B_{y 0} / B_{x 0}\right)$.

In order to compare the simulations directly with the satellite observations, let us consider such a virtual probe that is located at a spatial point $(x, y, z)$ in the plasma sheet. For simplicity, we assume that the probe does not move in spite of the progress of time as in the previous paper. Then, the virtual probe can readily detect the temporal changes of plasma quantities at its location on the basis of the simulation 

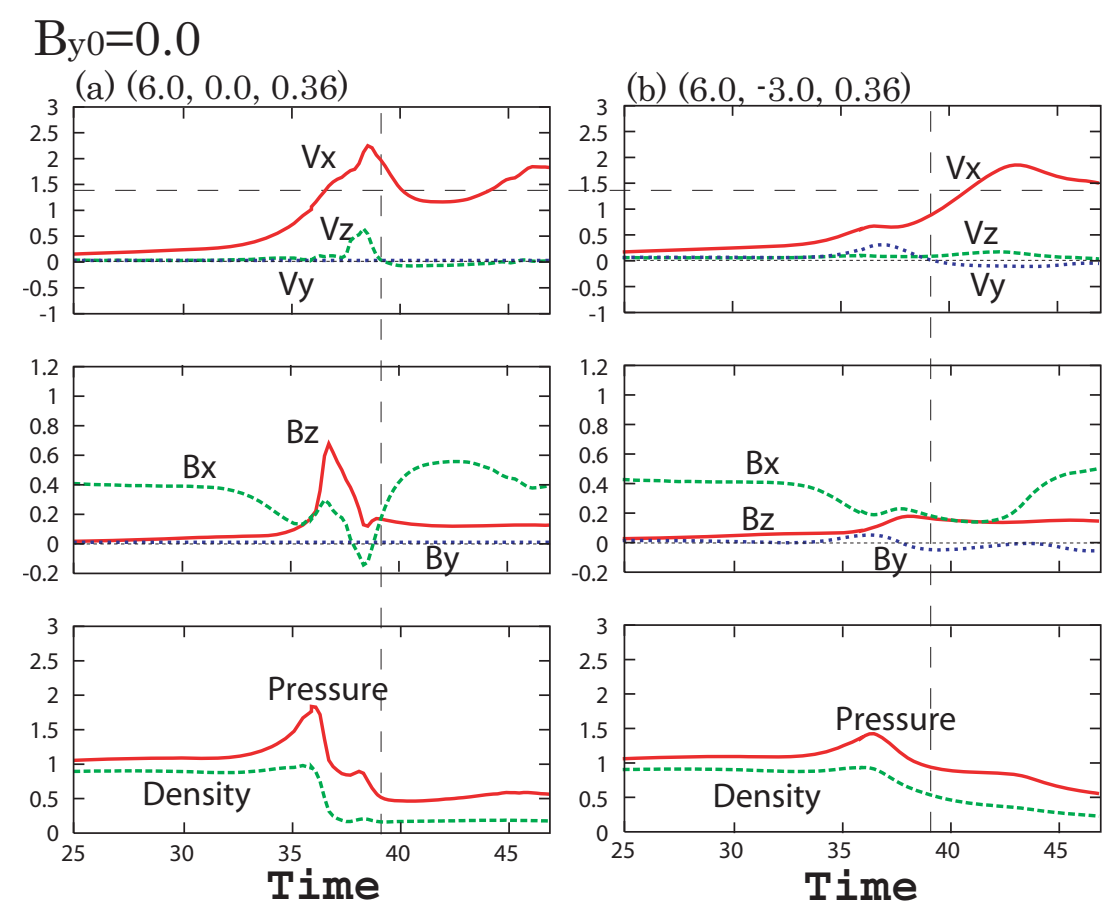

$\mathrm{By} 0=0.2$
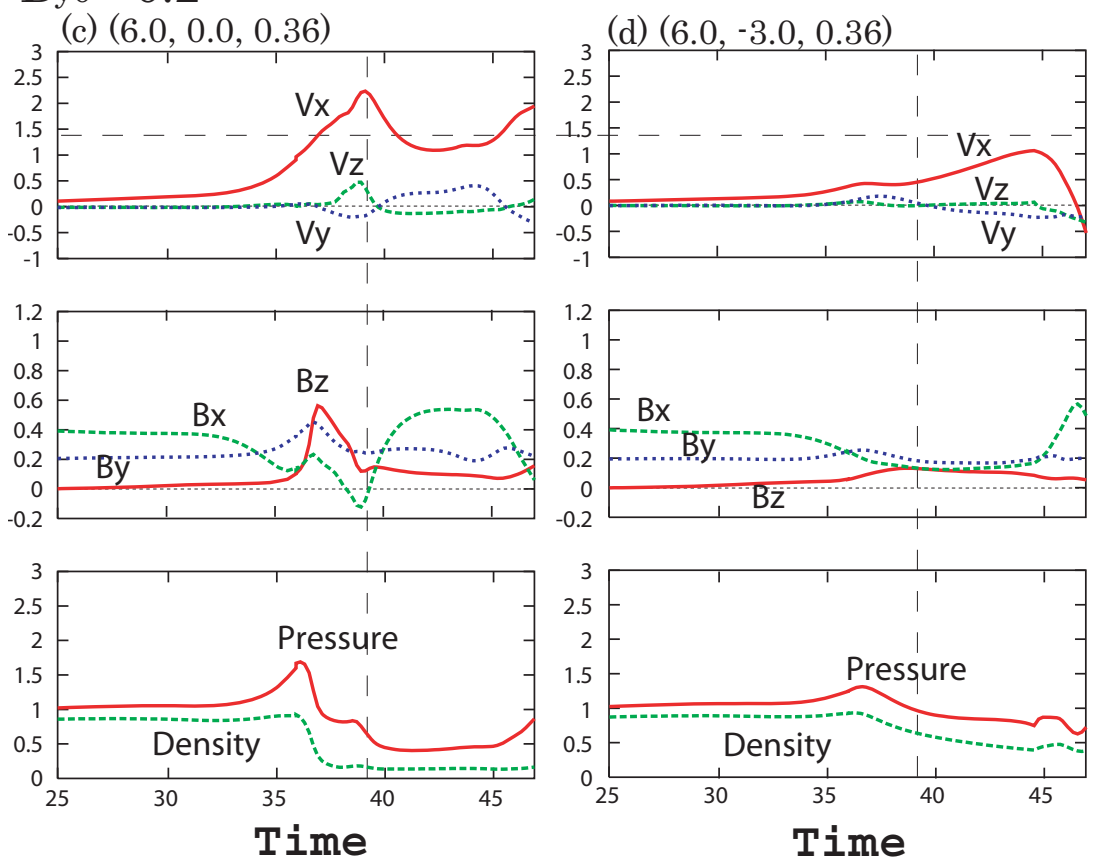

Fig. 4. Time profiles of $\mathbf{V}, \mathbf{B}, P, \rho$ detected at different positions in the y-direction, $(6.0,0.0,0.36)$ (left panels) and (6.0, -3.0, 0.36) (right panels) in our simulation domain in the case of $B_{y 0}=0.0$ (top panels) and $B_{y 0}=0.2$ (bottom panels).

results. Figure 4 shows the time variations of three components of flow velocity $\left(V_{x}, V_{y}, V_{z}\right)$, three components of magnetic field $\left(B_{x}, B_{y}, B_{z}\right)$, and plasma pressure and plasma density detected by two virtual probes located near the center of the fast flow channel $(6.0,0.0,0.36)(\mathrm{a}, \mathrm{c})$ and near the limb of it $(6.0,-3.0,0.36)(b, d)$. The top panels (a) and (b) show those in the case of $B_{y 0}=0.0$, while the bottom ones (c, d) show those in the case of $B_{y 0}=0.2$. These positions of virtual probes are indicated in Fig. 3. Vertical dotted lines in Fig. 4 indicate the time $T=39$, and horizontal dotted lines indicate the earthward flow speed 1.4, which is shown as the isosurface in Fig. 3. These two probes are located at 
different positions in the y-direction, and this difference drastically changes the time variations observed by them in spite of the same event.

In the case with no shear magnetic field $\left(B_{y 0}=0\right)$, the probe near the center of the fast flow channel in the ydirection (i.e. $y=0$ ), which is inside of the fast flow region at $T=39$ as shown in Fig. 3, observes rapid enhancement of $V_{x}$, large enhancement of $B_{z}$ and large rapid drop of plasma density as shown in Fig. 4a. On the other hand, the virtual probe near the limb of the fast flow channel in the y-direction (i.e. $y=-3.0$ ) observes gradual enhancement of $V_{x}$, small enhancement of $B_{z}$ and gradual decrease of $N$ as shown in Fig. 4b.

In the case with shear magnetic field $\left(B_{y 0}=0.2\right)$, the virtual probe near the center of the fast flow channel (Fig. 4c) observes very similar profiles to those in the case with no shear field in Fig. 4a. On the other hand, the virtual probe near the limb of the fast flow channel in the case with shear field observes significantly different variations in $V_{x}$ and $B_{x}$ from those in the no shear case, that is, rapid drop of earthward flow velocity $\left(V_{x}\right)$ and rapid recover of earthward component of magnetic field $\left(B_{x}\right)$ just before $T=45$ in Fig. $4 \mathrm{~d}$. The reasons of the observations of these variations are that the virtual probe suddenly goes out from the fast flow channel at $T=45$ due to the thinning of the fast flow channel and the deflection of the channel in the shear field direction. The thinning of the fast flow channel at the position far from the tail axis in the y-direction is usually very gradual in the case with no shear field, so that the virtual probe observes gradual long enhancement of $V_{x}$ as shown in the Fig. 4b. Considering the shear field, however, the virtual probe even at the same observation position is derailed from the fast flow channel due to the deflection of it as shown in the Fig. $4 \mathrm{~d}$ and Fig. $3 \mathrm{~b}$.

In Fig. 4b, c, d, the bipolar type variations in $V_{y}$ are observed during $V_{x}$ enhancement. The absolute $V_{y}$ value increases in the region of BBF interaction with the surrounding plasma (the interaction region), $V_{y}$ reaches its first extreme near the front, then decreases, changes its sign and reaches the second extreme somewhere in the BBF trailing part. These variations are also qualitatively consistent with actual satellite observations (Dmitrieva, 2008). The shear flow speed $V_{y}$ becomes larger as the dawn-dusk extent of the reconnection region becomes smaller, since the y-directional changes in quantities become larger. In the present study, we take a very long $k_{y}=8$ in Eq. (4), which makes the dawn-dusk extent of the reconnection region become very large, so that $V_{y}$ becomes small.

Let us compare with GEOTAIL observations (Fig. 1). The long enhancement of $V_{x}$ and the rapid decrease of $N$ are quite similar in Figs. 1a and 4a. The profiles of variations of magnetic field components are similar in these two observations, although the variations of them in Fig. 1a are small probably due to the observation near the reconnection region. On the other hand, the rapid decrease of $V_{x}$ and gradual decrease of $N$ are similar in Figs. $1 \mathrm{~b}$ and $4 \mathrm{~d}$. The variations of mag- netic field components are similar in these two observations, although the variations of them in Fig. 1b are large probably due to the observation position at far position from the reconnection region.

\section{Conclusions}

In this paper, the spontaneous fast reconnection model was applied to the earthward fast flow events observed in the nearEarth plasma sheet. The 3-D plasma configurations found in the present simulation considering shear magnetic field are summarized as follows: (1) The fast flow channel deflects in the direction of shear field. (2) The plasmoid is formed ahead of the fast reconnection jet and develops in three dimensions, and the plasmoid cross-tail size in the y-direction in the case with shear magnetic field almost the same as that in the case of no shear magnetic field.

On the basis of the simulation results, virtual probes were located in the plasma sheet, so that they could directly observe the temporal variations of plasma quantities. The virtual probe located at far position from tail axis in the negative $y$-direction easily goes out from the fast flow channel in the shear case, where it usually observes long and small enhancement of earthward fast flow due to the gradual thinning of the fast flow channel in the no-shear case. Considering the shear magnetic field, the rapid decrease of earthward fast flow as shown in Fig. 1b was able to be reproduced.

In summary, we have demonstrated that the satellite observations of the earthward fast flow event are generally consistent with the results caused by the fast reconnection mechanism. In fact, the virtual probe observations in the simulation domain could well explain different behavior of fast flows observed by GEOTAIL satellite.

Acknowledgements. This work was supported by Grant-in-Aids for Creative Scientific Research "The Basic Study of Space Weather Prediction" (17GS0208, Head Investigator: K. Shibata) from the Ministry of Education in Japan, Mitsubishi Foundation, RISH of Kyoto University, and Solar Terrestrial Environment Laboratory of Nagoya University. The computer program was tested and run at the Computational Centers of Nagoya and Kyoto University. Geotail magnetic field and plasma data were provided by $\mathrm{T}$. Nagai and Y. Saito through DARTS at Institute of Space and Astronautical Science, JAXA in Japan.

Topical Editor R. Nakamura thanks two anonymous referees for their help in evaluating this paper.

\section{References}

Angelopoulos, V., Baumjohann, W., Kennel, C. F., Voroniti, F. V., Kivelson, M. G., Pellat, R., Walker, R. J., Luhr, H., and Paschmann, G.: Bursty bulk flows in the inner central plasma sheet, J. Geophys. Res., 97, 4027-4039, 1992.

Dmitrieva, N. P.: Cross-tail velocity component in the plasma sheet fast flows, Ann. Geophys., 26, 1597-1604, 2008, http://www.ann-geophys.net/26/1597/2008/. 
Kondoh, K., Ugai, M., and Shimizu, T.: Three dimensional computer simulation of magnetic loop associated with magnetic reconnection, Adv. Space Res., 37, 1301-1304, 2006.

Kondoh, K. and Ugai, M.: Numerical studies on three-dimensional earthward fast plasma flows in the near-Earth plasma sheet by the spontaneous fast reconnection model, J. Geophys. Res., 113, A03S07, doi:10.1029/2007JA012707, 2008.

Lui, A. T. Y.: A Multiscale Model for Substorms, Space Sci. Rev., 95, 325-345, 2001.

Ohtani, S.-I., Shay, M. A., and Mukai, T.: Temporal structure of the fast convective flow in the plasma sheet:Comparison between observations and two-fluid simulations, J. Geophys. Res., 109, A03210, doi:10.1029/2003JA010002, 2004.

Ono, Y., Inomoto, M., Ueda, Y., Matsuyama, T., and Murata, Y.: Fast compression of a current sheet during externally driven magnetic reconnection, Earth Planets Space, 53, 521-526, 2001.

Petkaki, P. and Freeman, M. P.: Nonlinear dependence of anomalous ion-acoustic resistivity on electron drift velocity, Astrophys. J., 686-693, 2008.

Schodel, R., Baumjohann, W., Nakamura, R., and Mukai, T.: Rapid flux transport and plasma sheet reconfiguration, J. Geophys. Res., 106, 8381-8390, 2001.

Sergeev, V. A., Sauvaud, J.-A., Popescu, D., et al.: Multiplespacecraft observation of a narrow transient plasma jet in the Earth's plasma sheet, Geophys. Res. Lett., 27, 851-854, 2000.

Sergeev, V. A., Kubyshkina, M. V., Liou, K., et al.: Substorm and convection bay compared: Auroral and magnetotail dynamics during convection bay, J. Geophys. Res., 106, 18843-18855, 2001.

Shiokawa, K., Baumjohann, W., and Haerendel, G.: Braking of high-speed flows in the near-Earth tail, Geophys. Res. Lett., 24, 1179-1182, 1997.
Treumann, R. A.: Origin of resistivity in reconnection, Earth, Planets Space, 53, 453-462, 2001.

Ugai, M.: Self-consistent development of fast magnetic reconnection with anomalous plasma resistivity, Plasma Phys. Controlled Fusion, 26, 1549-1563, 1984.

Ugai, M.: Global dynamics and rapid collapse of an isolated current-sheet system enclosed by free boundaries, Phys. Fluids, 29, 3659-3667, 1986.

Ugai, M.: Computer studies on development of the fast reconnection mechanism for different resistivity models, Phys. Fluids B, 4, 2953, doi:10.1063/1.860458, 1992.

Ugai, M.: Computer studies on the spontaneous fast reconnection model as a nonlinear instability, Phys. Plasmas, 6, 1522-1531, 1999.

Ugai, M. and Kondoh, K.: Computer studies on the spontaneous fast reconnection evolution in various physical situations, Phys. Plasmas, 8, 1545-1552, 2001.

Ugai, M., Kondoh, K., and Shimizu, T.: Computer simulations on three-dimensional magnetic loop dynamics by the spontaneous fast reconnection model, Phys. Plasmas, 10, 357-363, 2003.

Ugai, M., Kondoh, K., and Shimizu, T.: Computer studies on the three-dimensional spontaneous fast reconnection model as a nonlinear instability, Phys. Plasmas, 11, 1416-1423, 2004.

Ugai, M., Kondoh, K., and Shimizu, T.: Spontaneous fast reconnection model in three dimensions, Phys. Plasmas, 12, 042903, doi:10.1063/1.1883181, 2005.

Ugai, M. and Zheng, L.: Parametric studies on traveling compression regions observed in the Earth's magnetotail, Phys. Plasmas, 13, 062906, doi:10.1063/1.2212827, 2006. 